\title{
Smart-BIM (Building Information Modeling)
}

\author{
B. de Vries $^{1 *}$, E. Allameh ${ }^{1}$, and M. Heidari Jozam ${ }^{1}$ \\ ${ }^{1}$ Department of Built Environment, Eindhoven University of Technology, Eindhoven, \\ Netherlands \\ *Corresponding author (B.d.Vries@tue.nl)
}

\begin{abstract}
Purpose After a long period of international research and development, BIM has become mature. Many tools support the BIM process, or at least they claim. BIM not only offers opportunities for the Architectural Engineering and Construction industry, but also for the client. In this paper we don't focus on the professional client, but on the client of a building assignment that act as the end-user. Involvement of the end user in the design process has been advocated by many scholars and designers, but has so far only marginally been adopted in practice. The importance of user participation is demonstrated by the lack of success of smart technologies in new housing or in renovation. Particularly elderly people resist accepting these technologies in their home environment, although they could benefit from these technologies to improve comfort and health care. As a result of poor understanding of these new technologies by both designers and end users, researchers observe that there is a mismatch between user demands and smart technology usability. Hence, this paper is an attempt to improve the role of users in the design process in two ways. Firstly, by adding the missing components of smart technology to current BIM model libraries. Secondly, by developing a virtual model in which users can interact with the smart technologies and configure their preferred layout. The final results are interesting not only for technology developers but also for housing designers who aim at improving the quality of life in future housing for aging society. Method For a better understanding of BIM, a historical perspective is taken in this paper. The initiatives from different research institutes are discussed and how they affected each other. The up-take by the software industry is highlighted and their delicate relationship with science. In today's design process BIM systems support spatial design that is accommodate by smart technology. Usually this smart technology is added after the spatial design in the final design stage by the installations expert. In our research we want to turn this process around; the smart technologies are accommodated by spatial design. Therefore we develop a design system with a library of smart components such as smart wall, smart kitchen and smart furniture. The difference between smart technologies and standard building components is that smart technologies interact with the building users. BIM allows for realistic visualization of designs in an early stage. In our prototype system, clients are presented a virtual space with a wide range of smart technologies. After being introduced to these technologies, the client expresses how these will fit within his/her activities. Following he/she can experience in the virtual model how smart technologies react when activities are executed. Results \& Discussion A prototype system is presented that allows clients such as elderly to experience smart technologies. In contrast with traditional design it does not start from the spatial layout but from the activities that should be accommodated supported by smart technologies. We expect that fundamentally different layout will emerge from this approach. Although no experimental data are available yet, some first experiences will be discussed.
\end{abstract}

Keywords: BIM, smart technology, client support

\section{INTRODUCTION}

After a long period of international research and development, BIM has become mature. Many tools support the BIM process, or at least they claim. BIM not only offers opportunities for the Architectural Engineering and Construction industry, but also for the client. In this paper we don't focus on the professional client, but on the client of a building assignment that act as the end-user. Involvement of the end user in the design process has been advocated by many scholars and designers, but has so far only marginally been adopted in practice. The importance of user participation is demonstrated by the lack of success of smart technologies in new housing or in renovation. Particularly elderly people resist accepting these technologies in their home environment, although they could benefit from these technologies to improve comfort and health care. As a result of poor understanding of these new technologies by both designers and end users, researchers observe that there is a mismatch between user demands and smart technology usability.

In today's design process BIM systems support spatial design that is accommodate by smart technology. Usually this smart technology is added after the spatial design in the final design stage by the installations expert. In our research we want to turn this process around; the smart technologies are accommodated by spatial design. Therefore we develop a design system with a library of smart components such as smart wall, smart kitchen and smart furniture. The difference between smart technologies and standard building components is that smart technologies interact with the building users.

BIM allows for realistic visualization of designs in an 
early stage. In our Smart Design system, clients are presented a virtual space with a wide range of smart technologies. After being introduced to these technologies, the clients express how these certain technologies will fit within their scheme of daily activities and give their requirements and feedbacks while performing tasks in the virtual model.

In our view, Smart-BIM is composed of Smart Design system using Smart technologies. Hence, the outline of this paper is as follows. First, we describe what the features of Smart technologies are and how they are related to existing BIM components. Following we present the Smart Design system functionality and how it can support the client to experience interaction with the smart technologies. Finally, we draw conclusion on the consequence of Smart-BIM for the design process and the building industry.

\section{SMART TECHNOLOGIES}

BIM is not possible without standardization of building components. Many building component libraries have been developed for different aspects of the building design, such as spatial design, structural design, installations, etc. Often these libraries are included in Architecture, Engineering and Construction (AEC) tools, or they are provided by product suppliers. To support the quest for data exchange models between different AEC tools, standardization efforts have focussed on building components. The most widely spread ISO certified building component library is the Industry Foundation Classes (IFC) standard. Building component libraries like IFC have developed from traditional catalogues of building products. The main challenge is to define a parameterized geometrical description and to define the additional attributes for material, cost, etc. Building components libraries are intensively used in the AEC industry today and thus have proven their success. However, since they are based on traditional building components, they also prohibit fast adoption of new building components.

Smart objects contain their own functions in their property set to interact with users and other objects. Defining the property set of these smart objects needs an understanding of their function in real situations. In the following, three types of smart objects, their functions and features are introduced:

Smart Kitchen Table is a normal table with supplementary attributes of:

- Touch Screen Surface which allows the size of hot zones and temperature to be adjusted by user. It allows the user to browse the internet on the surface.

- Multimedia networking which let different electrical appliances communicate with each other. Internet, recipe database, television as well as other building services can be operated and controlled from it ${ }^{1}$.

- Dynamic Table Top Interface made of wireless power which makes it possible to have no preset cooking zones. Cooking takes place anywhere, anytime on the table. Energy will follow the devices as they move around the surface, and so the interface will be displayed on the table top surface ${ }^{2}$.

Smart Wall combines the concept of both TV and Computer together integrating in a wall. This revolutionary piece of equipment can be used in either a fixed or temporary environment, allowing the user to create an interactive working and living space in almost any location. The objective of this Wall is to serve the following attributes:

- Changeable scenery system which creates different scenery on the wall by adding the elements of entertainment and sensitivity to the wall.

- Interactive electronic surfaces on the wall represented by a touch-sensitive information device.

- Internet connected system enables several teleactivities such as tele-educating, tele- caretaking, tele- team working. The goal is to support two or more persons, in parallel or sharing the whole display space ${ }^{3}$. It also covers virtual communications relying on more natural gestures.

- Environmental control system which is connected to smart context. Hence, Smart Wall environment reacts to users' function by adapting the HVAC conditions and natural/artificial light for a personalized room ambience ${ }^{4}$. Also it is connected to the other home digital devices; users can manage other home appliances through the Smart Wall.

Smart Furniture has different user interface and interaction styles from current furniture and computers which have standard desktop PC, single user display, keyboard, and mouse. Computers are going to be embedded in tables, seats and mobile devices. They are also connected to each other and to the whole Home Network. So the data can easily transfer among different furniture. The common features of Smart furniture can be introduced as:

- Flexible and movable,

- Intelligent and aware of users need and preferences, due to several sensors,

- Responsive to users activity, due to being programmable,

- Interactive, due to equipping with touch screen surfaces or LCD,

- Multifunction, due to supporting different virtual activities, entertainment activities, relaxing, work related activities also managing other device and environmental conditions, such as HVAC $^{5}$. 
All the objects and technologies inside the Smart Home support people carrying out their everyday activities, tasks and rituals in an easy, intelligent and interactive way. Accordingly, the difference between a 'normal' library component and a smart library component is the interaction between the component and the building users. If we take as an example the ifc-Wall Standard Case and its property set, this wall type will contains a geometry description and a list of properties such as: Acoustic Rating, Fire Rating, Combustible, Surface Spread of Flame, etc. The wall geometry and properties are determined in a long standardization process by the most common wall types that are found in today's building sector. In case of a smart wall, there are hardly any precedents. The definition of a smart wall should not only describe the geometry and material, but also the interaction with its intended users. This description can be static, but then the added value in the design process is limited. We think that designing a smart building requires interactive building components. Interactive building components respond to touch, remote control, motion detection, or whatever method is used to interact. Interactive building components are often integrated components consisting of constructive parts and embedded technologies. These embedded technologies can range from LCD screens to micro sensors.

Digital representations of interactive systems are not entirely new. On the internet we can find many examples (often implemented in Flash) of commercial products that one can view and virtually operate by clicking on buttons or hot spots ${ }^{6}$. This type of information sharing is not only more attractive but also gives a better sense of reality, then plain technical data. In this example, interaction is established through mouse-clicks. For more realistic evaluation of a virtual building model, the smart building components should be able to receive input from multiple sensors and to act accordingly. Because today information about multi-sensory interaction is still rare, standardization of these new technologies is a long shot. But for existing interface technologies like remote control, implementation of a smart building component is very well possible.

Technically, digital building component libraries need to be extended with interactive $2 \mathrm{D}$ or $3 \mathrm{D}$ models to turn these into smart components. In current digital libraries, we can also find multiple representations for the same product. Multiple representations have proven to be useful for different levels of detail and for hiding irrelevant information. An interactive model can be seen as yet another view on the same product. An example of interactive digital model of a smart wall is presented in Figure 1.

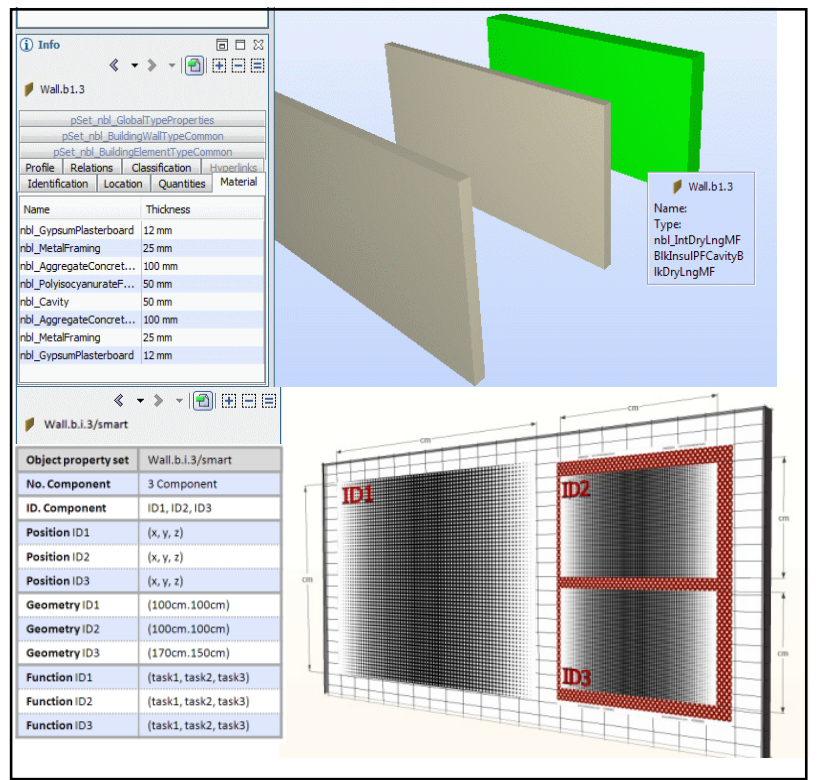

Fig.1. Interactive digital model of a smart wall: Each smart object contains several smart components with a property set.

A smart object consists of several smart components with a property set that specifies its capabilities to respond to user activities. Figure 1 shows a smart wall with three interactive surfaces as smart components. A smart wall 'senses' the activities that are executed and it will act accordingly for instance by switching on screens for different purposes such as tele-communication, tele-shopping, entertainment, etc.

\section{SMART DESIGN}

As any smart home will be eventually used by end users, providing a method to enhance user participation for interaction manipulation is indispensable. Our Smart Design system applies the user-centered approach to let users interact with the objects and experience smart living in a new home setting. Using VR technology, the platform is capable of visualizing smart technologies and performs real-time interactions with the home. This Smart Design system is based on a task-based model in which end users interact with the system and experience how smart objects respond to typical domestic activities.

This proposed design system aims to collect information on people's appreciation of smart technologies. The system not only supports design of home interiors but also elicitation of activities that a user wants to perform within the contextual conditions. This integration aims to facilitate both end-users and designers to understand how the users' activity will be accommodated in the given home setting.

There are other researches which aim to simulate and predict occupants' activities in the given building and evaluate the building performance including evacuation, circulation, building control system ${ }^{7}$. 
While many of them focus on office environments like Tabak' s research ${ }^{8}$ and non-smart environments, our proposed design system can be used to experience not only how a smart building will look like but also how users will interact with such a kind of environment based on activity contexts. It improves users' understanding on the design, help them specify their activities in the given new home setting and increase their involvement in the communication with designers. The system also let designers evaluate the design (in terms of spatial properties and technology functions) and collect users' feedback of the design by a task-based interaction between user and building.

Since the relation between the user and the building is mediated through the spaces where the activities take place, human-space interaction plays a key role in Smart Design system. Behaviour of the users and their response to technology is critically dependent upon the environment and context in which information is presented or requested. The most popular assessment instruments in use today for studying the activities of people in natural settings are self report recall surveys, time diaries, direct field observation, and experience sampling ${ }^{9}$. Developing daily activity specifications could be helpful method to have a more realistic interaction. Oxman et $\mathrm{al}^{10}$ introduced three design paradigms to induce interaction process with virtual environment: task-based design, scenario-based design and performancebased design. Implementing each of these paradigms enhances users experience in virtual environment and improves the human sense of "being there"11. While scenario-based design and performance-based design are based on specific predefined situations, task-based results could be closer to users' real reactions. Task-based process enables users to imagine the scheme of their daily activities in the task context and react accordingly. It also enables context reaction toward users' interaction and functions as found in physical smart space. Finally, an improved understanding of smart technology usage is expected from both end users and designers through this task-based interaction.

Applying this Smart Design system needs Smart BIM, that is, a model that includes smart objects and users tasks. Smart objects are part of a building model. A smart object consists of one or more smart components that have specific capabilities. Examples of HasCapabilities are: Displaying, Heating, Lighting, etc. A user will execute many tasks in the home environment. Each task is determined by the combination of a zone in the building model and an activity from the activity list. The activity list contains two types of activities: (1) Main activity and (2) Secondary activity. A task always contains one main activity (e.g. cooking) and may contain one or more secondary activities (e.g. TV watching). An activity has one or more NeedCapabilities. Examples of NeedCapabilities for activity cooking are: Baking, Recipe displaying, Air cleaning, etc.

The structure of this Smart Design system is presented in Figure 2.

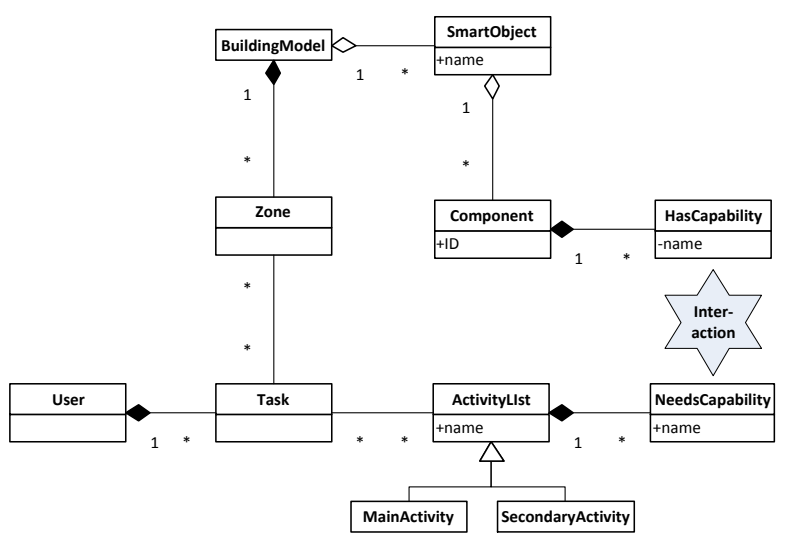

Fig.2. Smart Design data model

The interaction between user and its smart home is established through matching the NeedCapabilities with the HasCapabilities. In our prototype Design Systems this matching procedure is a simple rulebased system, because it aims to experience smart objects, but avoid too much complexity for the user. In reality, however this matching process is performed by an intelligent home system that lets smart technologies communicate with each other.

In a smart design session, the designer will first create the home interior like with any CAD program but with use of smart objects. After the client is satisfied with the spatial design, the designer will create specific zones in the home and enter some general information about the client. In discussion with the client, the designer determines the tasks that the client wants to perform in his/her new home. For each task, the client makes a selection from the activity list and a selection from the available zones. Then the smart design is ready for design experience by the client. The client is requested to navigate through the digital building model. At any spot in the home, he/she can execute a task. Therefore, he/she can use a virtual mobile phone to select a predefined task. After task selection, the smart objects will respond. The type of response is determined by the rule-based system that is called upon by the smart design system.

As an example, the user will navigate to the smart kitchen table (Figure 3). In the zone, the virtual mobile phone will pop up. After selection of main activity= cooking and secondary activity= watching TV, the kitchen table will present a cooking area. At the same time, one of the TV screens nearby scans the TV channels for the latest news and another one shows a web site with cooking recipes for users' diet. Because the person is an elderly, the floor sur- 
face will be sensible for falling and the lighting is set to a high level for a better vision.

In discussion with the designer, the client expresses his/her appreciation, misunderstanding, disapproval, etc, and makes suggestions for improvement of the design.

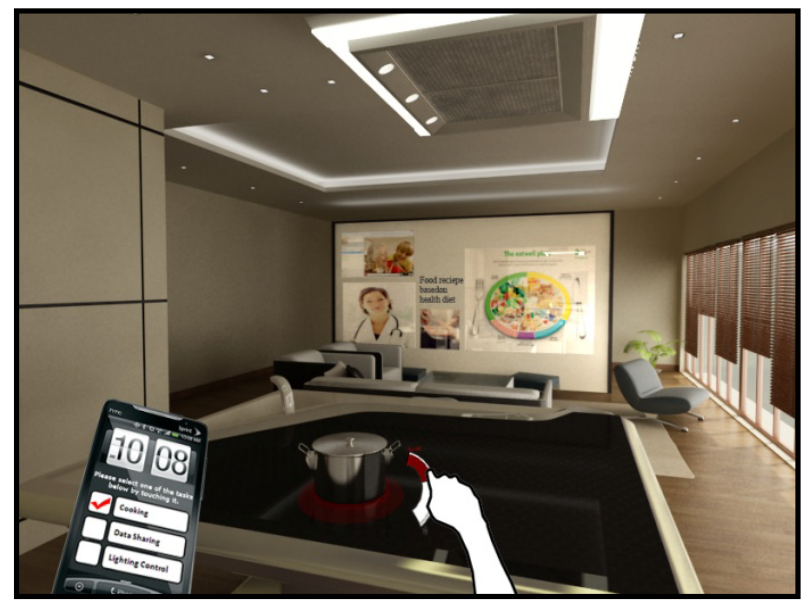

Fig.3. Smart Design prototype interface

\section{CONCLUSION}

We believe that smart-BIM will change the building design and the building process. Only if smart objects are part of the CAD libraries, smart objects will become a natural choice in the design process. Like normal building objects, smart object are a representation of reality. Although today computer systems can do wonderful things, the perception of space and material through virtual models is limited. Likewise the perception of smart objects will be limited but nevertheless very valuable. Experiencing real-time response in a virtual model adds to the understanding of the smart home environment. The building process will change because in a smart design process, it is impossible to design without the involvement of the client/user. Participatory design has not been very successful so far, but for smart design it is a precondition. At various stages of the design process, user's acceptance needs to be evaluated. User involvement through shared virtual models is possible today and may provide designer and engineer with much new information upfront.

We imagine that the smart design system can be used for other purposes, such as: measuring user preferences, space utilization analyses, user behavior research, etc. In our future research, we will investigate these possibilities and extend the library of smart objects.

\section{References}

1. Stackelbeck, N., "How will we be cooking and living tomorrow? Kitchen Concept 2015 provides inspiration for future developments", Kirchlengern/ Germany, Available at: http://www.hettich.com/id_EN/news/press/press- de-

tail.html?tx_hettich_pipresse[action]=detail\&tx_hettic h_pipresse[controller]=Presse\&tx_hettich_pipresse[ news] $=100 \& \mathrm{cHash}=0 \mathrm{a} 2916 \mathrm{ec} 3 \mathrm{ab} 5 \mathrm{f}^{7} 9 \mathrm{bd} 3683 \mathrm{e} 7 \mathrm{bec}$ 4801aa, 2009. (accessed 22 February 2012)

2. Philips Designers, "Philips Simplicity Event 2008 Green Cuisine Concept", available at: http://www.newscenter.philips.com/main/standard/a bout/news/press/20081015_simplicity_event_green _cuisine.wpd, 2008. (accessed 22 February 2012)

3. Fraunhofer Institute, "Roomware", available at: http://www.ipsi.fraunhofer.de/ambiente/english/proje kte/projekte/roomware.html/, 2004. (accessed 22 February 2012)

4. Philips Designers, "daylight concept", available at: http://www.newscenter.philips.com/main/standard/a bout/news/press/20071023_simplicity_concept_coll ection.wpd, 2007. (accessed 22 February 2012)

5. Allameh, E., Heidari Jozam, M., de Vries, B., Timmermans, H,. Beetz, J., "Smart Home as a smart real estate, A state of the art review", 18th Annual European Real Estate Society Conference, Eindhoven, the Netherlands, 2011.

6. Home auto, available at: http://www.interinter.com/haihouse.swf

Hettich House, available at: http://www.hettich.com/discoverhettich/de/LivingRoo m.html

LGHomeNet, virtual experience space, available at: http://www.lghomnet.com/homnet/exper/exper.html

7. Shen, W., Shen, Q., Sun, Q., "Building Information Modeling-based user activity simulation and evaluation method for improving designer-user communications", Automation in Construction, Vol 21, pp. 148-160, 2012.

8. Tabak, V., de Vries, B., "Methods for the prediction of intermediate activities by office occupants", Building and Environment, p.45, 2010.

9. Lertlakkhanakul, J., Won Choi, J., Yun Kim, M., "Building data model and simulation platform for spatial interaction management in smart home", Automation in Construction, Vol. 17, pp.947-957, 2008.

10. Oxman, R., Palmon, O., Shahar, M., Weiss, P.L., "Beyond the reality syndrome: designing presented in virtual environments", in: $\mathrm{H}$. Orbak (Ed.), Proc. ECAADE 2004, Copenhagen, pp. 15-18, 2004.

11. Oxman, R., "Design paradigms for the enhancement of presence in virtual environments", in: collaborative design in virtual environments, ISCA 48, Springer, pp. 41-49, 2011. 